\title{
La bruyère arborescente (Erica arborea), un ligneux bien valorisé par des chèvres laitières en zone méditerranéenne française
}

\author{
JP Goby, JJ Rochon \\ Institut Universitaire de Technologie Chemin de la Passio Vella - 66860 Perpignan Cedex, France
}

L'abandon des suberaies du massif des Albères au sud des Pyrénées Orientales en France a permis l'installation d'un maquis dense constitué essentiellement de bruyère arborescente (Erica arborea). Face à cette situation, différents partenaires (bouchonniers, propriétaires et services publics) ont su unir leurs moyens pour assurer la remise en état de la suberaie et limiter les risques d'incendie induits par l'embroussaillement. Le développement d'élevages de chèvres, animaux particulièrement adaptés à la consommation de ligneux, est un moyen de contrôler la végétation tout en ayant une fonction de production capable d'assurer un revenu à l'éleveur.

Les analyses chimiques de la bruyère arborescente montrent des taux élevés en lignine $(30 \%)$ et en tannins $(11 \%)$ qui induisent une faible utilisation digestive des fibres (la dégradation ruminale $48 \mathrm{~h}$ de la matière sèche est de $38,8 \%$ dont $28,6 \%$ avant $8 \mathrm{~h}$ caractérisant la disparition de la phase soluble). Malgré ces faibles valeurs nutritives, cet arbuste est omniprésent dans la ration prélevée sur parcours (entre 20 et $80 \%$ de la matière sèche ingérée) par des chèvres à haut potentiel laitier ( $800 \mathrm{~V} / \mathrm{an})$.

Des mesures de l'évolution des paramètres ruminaux $\left(\mathrm{pH}, \mathrm{NH}_{3}, \mathrm{AGV}\right)$ montrent que l'ingestion de bruyère arborescente induit, très rapidement, dès la sortie sur parcours à 12 heures, une évolution significative des 3 paramètres (chute du taux d'ammoniaque et du $\mathrm{pH}$ et augmentation des acides gras volatils). Un suivi de la dégradabilité ruminale 48 heures d'un foin dans deux régimes alimentaires $(100 \%$ foin et $50 \%$ foin $+50 \%$ bruyère) montre que le régime foin + bruyère a une influence positive hautement significative sur la dégradabilite 48 heures de la matière sèche du foin $(57,3 \%$ contre $51,0 \%$ ). Bien que l'analyse fourragère suggère une faible valeur nutritive, la bruyère arborescente semble apporter un élément capable de stimuler l'activité cellulolytique du rumen. Le ralentissement du transit induit par l'ingestion de tissus lignifiés peut aussi expliquer une meilleure digestion ruminale des fibres cellulosiques.

La proportion de feuilles dans la biomasse épigée de la bruyère est fortement réduite par des pâturages fréquents. La gestion des parcours dans ces zones doit tenir compte à la fois de la lutte contre les incendies mais surtout de la régénération de la strate arbustive pour pérenniser le système.

Evolution de 3 paramètres ruminaux au cours de la journée.

\begin{tabular}{lcccccc}
\hline Heures & 8 & 10 & 12 & 14 & 16 & 18 \\
$\mathrm{pH}$ & $6,6 \pm 0,2$ & $6,6 \pm 0,2$ & $6,4 \pm 0,2$ & $6,0 \pm 0,2$ & $5,9 \pm 0,1$ & $6,0 \pm 0,1$ \\
$\mathrm{NH}_{3}(\mathrm{mg} / \mathrm{l})$ & $91,7 \pm 28,2$ & $105,4 \pm 30,0$ & $94,5 \pm 22,4$ & $61,8 \pm 19,9$ & $70,6 \pm 32,2$ & $70,1 \pm 38,6$ \\
$\mathrm{AGV}(\mathrm{mM} / \mathrm{l})$ & $66,0 \pm 11,6$ & $68,6 \pm 10,1$ & $71,1 \pm 12,0$ & $85,5 \pm 7,8$ & $90,6 \pm 8,7$ & $86,9 \pm 9,5$
\end{tabular}

